MORPHOLOGICAL ANALYSIS, DIFFUSION AND LOCK-OUT OF TECHNOLOGIES: FERROUS CASTING IN FRANCE AND THE FRG

Dominique Foray

CNRS, Lyon, France

Arnulf Grübler

International Institute for Applied Systems Analysis,

Laxenburg, Austria

RR-91-1

February 1991

Reprinted from Research Policy Volume 19, No. 6, December 1990.

INTERNATIONAL INSTITUTE FOR APPLIED SYSTEMS ANALYSIS

Laxenburg, Austria 
Research Reports, which record research conducted at IIASA, are independently reviewed before publication. However, the views and opinions they express are not necessarily those of the Institute or the National Member Organizations that support it.

Reprinted with permission from Research Policy Volume 19, No. 6, December 1990. Copyright (c)1990 Eisevier Science Publishers B.V. (North Holland)

All rights reserved. No part of this publication may be reproduced or transmitted in any form or by any means, electronic or mechanical, including photocopy, recording, or any information storage or retrieval system, without permission in writing from the copyright holder.

Printed by Novographic, Vienna, Austria 


\section{Foreword}

Dominique Foray and Arnulf Grübler address important, but as yet unresolved, conceptual and methodological questions in the area of technological change and diffusion research. What, for example, constitutes a formal framework for the definition of new technologies and what are the conditions that allow for successful experimentation and market introduction of initially inferior technologies? Their approach to providing an answer to some of these questions starts with a definition of the object that is diffusing, its interaction with the technological environment in which it is embedded, and the transformation it undergoes during its diffusion. To this end, the authors introduce the concepts of technological neighborhood and distance derived from a morphological analysis of the entire technological space for a particular function. This then serves as a methodology for defining competing technological routes, illustrated for the case of non-ferrous casting processes. The authors show the fruitfulness of their approach not only to better conceptualize and model discontinuities in diffusion trajectories, but also to capture the critical importance of small initial market niches, that provide the ground for experimentation and learning inside the industry, before diffusion actually takes place. This mechanism allows a new technology, initially inferior to its competitors, to escape from a lock-in situation and become competitive; if successful it will also diffuse inside the market at large.

Foray and Grübler conclude this analysis by developing a scheme - an evolutionary tree - to describe comprehensively the changing nature and characteristics of technologies during diffusion. The object diffusing is different at each phase of the process. It undergoes a whole series of evolutionary changes, transforms itself and thus widens its fields of application and mar-

kets. It is this intrinsically interrelated process of technological mutation (to use a Schumpeterian term) and interaction within the market environment that forms and shapes the diffusion processes and the eventual replacement 
of old by new. This process can be comprehensively described by the framework proposed by Foray and Grübler. The results of the study evolved out of a collaborative research effort between Centre National de la Recherche Scientifique (CNRS) and IIASA. This work will continue in the future with the objective of gaining a better understanding of the mechanisms and policies promoting technological change in energy systems as a response to evolving global environmental boundary conditions.

NEBOJŠA NAKIĆENOVIĆ

BO DÖÖS

Project Leader

Leader

Environmentally Compatible Energy Strategies

Environment Program 


\title{
Morphological analysis, diffusion and lock- out of technologies: Ferrous casting in France and the FRG *
}

\author{
Dominique FORAY \\ CNRS, Lyon, France \\ Arnulf GRÜBLER \\ IIASA, Laxenburg, Austria
}

Final version received January 1990

In this paper we will try to address technological diffusion and substitution from two methodological angles. The first consists in a complete and comprehensive morphological analysis (MA) of a set of (process) technologies for a particular industry. In the second part, we use the results of our MA of the technological trajectories in the casting industry, to analyse their diffusion in two countries, France and the FRG. With respect to the results of this work, we can express two observations. First, this case study will provide valuable insights on the conditions of exit from a "lock-in" situation. Second, the MA will permit us to avoid misinterpretation concerning the asymmetrical character and discontinuities of the diffusion trajectory of the technology under consideration.

I have often wondered why no economists or economic historian has ever tried to work out classifications like those for living organisms, related to the trees of evolution of techniques, and their embodiments in industries and commodities. As it is, our statistical knowledge of techniques is rather thin for the ambitious aim of a model.

J. Steindl [21]

\footnotetext{
* Financial support from the Centre National de la Recherche Scientifique (CNRS) and the International Institute for Applied System Analysis (IIASA) is gratefully acknowledged. An earlier version of this paper was presented at the International Conference on Diffusion of Technologies and Social Behaviour, 14-16 June 1989, Conference Center Laxenburg, Austria. We are indebted to two anonymous referees for helpful comments on this earlier version.
}

Research Policy 19 (1990) 535-550

North-Holland

\section{Introduction}

The historiography of technical progress $[14,16]$ has established that the process of technological diffusion is in itself also a developmental process. In other words, it is in its diffusion throughout the economy that a technology acquires its industrial properties, transforms itself, and widens the initial market in which it was adopted. On the basis of these dynamic properties of the diffusion process, some authors have been hasty in inferring the theoretical impossibility of formal representation, since the object of the diffusion is not the same at the beginning, in the middle, and at the end of the process. It appears to us, however, that the interest of a formal representation resides precisely in the possibility of periodizing the diffusion process, with the aid of criteria which can take into account the principal transformations of the technology under consideration. The diffusion process can thus be considered as a series of competitions at given times between a technology $(A)$ which is in the middle of a transformation and other technologies $(B, C, D)$ with respect to those functions that $(A)$ is successively able to assume. ${ }^{1}$ We may, intuitively, suppose that these successive competitions will occur in ever larger markets as $(A)$ progressively sheds its initial specialty. It is therefore possible to interpret the diffusion pattern characteristics of a given period on the basis of the

${ }^{1}$ A relatively similar purpose is presented by Cameron and Metcalfe [3]. 
manner in which competition developed throughout a previous period.

The first part of our paper consists therefore in a complete and comprehensive morphological analysis (MA) of a set of (process) technologies for a particular industrial activity, in our case ferrous casting. Through the MA approach proposed, we will be able to define the criteria of the periodization of the diffusion process of the technology under consideration. More generally, we intend to show the importance and fruitfulness of an explicit and formal methodology in defining the technologies competing/diffusing in a particular market, which by its comprehensive nature is not time or industry statistic dependent.

In the second part, we use the results of our MA of the technological trajectories in the casting industry, to analyse their diffusion in two countries, France and the FRG. We describe first the distinctly different patterns of the technological trajectories used in the industry of the two countries. We then continue to discuss the possible driving forces behind the "locking-out" of the gasifiable pattern process technology (GP process) in France and its diffusion in the FRG by means of standard diffusion methodology. ${ }^{2}$ On the basis of the MA we describe the diffusion of the GP process as proceeding via successively filling two market niches: first, small batch size production and later, following improvements in the technology, also mass-production of ferrous castings. In the case of the FRG we point out the extreme importance of the early start of the diffusion process of the GP technology inside the first market niche, which generates a process of accumulation

${ }^{2}$ The quantification of the diffusion trajectories will be based on a simple Fischer - Pry [4] type of technological substitution model.

${ }^{3}$ We are indebted to the theory of "lock-in" [1] for a clear understanding of the mechanisms (increasing returns to adoption) by which a technology overcomes its rival and then produces its own defense mechanisms in order to preserve its monopolistic situation position, even against inherently superior technologies. The principal sources of the increasing returns to adoption are: learning by using, network externalities, scale economies in production, informational increasing returns, technological interrelatedness and finally, the production of ad hoc evaluation norms. The two latter sources will allow us to more precisely explain the phenomena of the long-term maintenance of mature technologies. of knowledge and of learning (not the case in France) leading to the widening of this initial market niche.

With respect to the results of this work, we can express one analytical observation and one methodological observation. First, this case study will provide valuable insights on the conditions of exit from a "lock-in" situation. ${ }^{3}$ Second, the MA will permit us to avoid misinterpretation concerning the asymmetrical character and discontinuities of the diffusion trajectory of the GP process.

As in any analysis proceeding from the macro to the micro level, increasing detail and data intensiveness accompany diffusion research. The choice of the particular sector and the country examples considered to empirically illustrate our methodological propositions, was thus determined neither by the macroeconomic importance of the ferrous casting industry (in terms of employment or contribution to GNP) nor by the growth prospects of this particular industry branch. Instead, the possibility of carrying out a comprehensive morphological analysis in a well structured space of technological trajectories as well as the detailed preparatory work on the history and evolution of the innovations in this particular branch $[5,6]$ determined the choice of this particular case study. We maintain, however, that the type of approach proposed constitutes a first step towards a more thorough analysis of technological diffusion processes. As such, it may open the possibility of ultimately developing a taxonomy and classification of technologies and their diffusion processes, which we consider necessary for the advancement of the theoretical foundations and practical usefulness of diffusion studies.

\section{The morphological space of molding technology}

The first step in any analysis of technological diffusion and substitution processes is to define the technologies under consideration in a generic manner. In a best case situation, this is done based on expert knowledge, whereas in a worst case, the definition of technologies remains rather arbitrary, based on the disaggregation level available in industry statistics. Such a situation appears unsatisfactory both from the atheoretical nature of the 
approach as well as from the perspective of identifying possible technological routes which might emerge in the future or which have been "locked out" from diffusion in the past. The use of the morphological analysis (MA) appears to us to be capable of resolving these introductive difficulties, associated to every diffusion's analysis.

Morphology, as the "science of form", studies the structure or form of something. In the field of design, MA consists of the rigorous examination and evaluation of all possible alternatives to each structural part of a problem. This aims to give the optimum solution(s) by virtue of having considered every possibility. In the first section we attempt to use MA in order to build the morphological space of molding technology, i.e., to derive a complete description and taxonometric classification of the technologies used in the molding process.

\subsection{Morphological methods: principles and practice}

MA is a technique for identifying, indexing, counting and parametrizing a collection of all possible devices to achieve a specified functional capability $[11,20]$. An MA applies the following rules: well-structured problem, identification of the characteristic parameters, subdivision of each parameter into cases or "states" $p_{k}^{1}, p_{k}^{2}, p_{k}^{n}$, and identification of the various combinations. Besides, we use the following basic definitions: Morphological space $\left(p_{K}^{j}\right)$ consists of a set of discrete points or "coordinates", each corresponding to a particular combination of parameters. The space has as many dimensions as parameters. Morphological distance between two points in the space is the number of parameters differing from one another in two configurations. Morphological neighbourhood is a subset of points, each of which is morphologically close to the other. Technological breakthrough is achieved when a new configuration is obtained.

An MA starts with building a morphological space for a particular set of technologies or products, in order to understand and thus not to "miss" a technological route of possible future development. The morphological space is defined by any number of dimensions and subdivided into elementary spaces which show the "state" of technology under consideration.

\subsection{Building a morphological space for molding technology}

First, the problem to be solved (or the functional capability desired) must be stated with great precision; in our case, the problem consists of realizing ferrous metal products by a casting process (molding technology).

Second, relating to this definition, four characteristic parameters are identified:

$P_{1}$ : the nature of the pattern

$P_{2}$ : the nature of the mold cavity

$P_{3}$ : the stabilization force

$P_{4}$ : the bonding method

Third, each parameter can be subdivided into states.

$\left(P_{1}^{1}, P_{1}^{2}\right.$ : permanent or lost): The molding methods can be classified according to the nature of the pattern (form of product). In the first case (wooden and metal pattern), the pattern is used for a large number of castings. In the second case (lost wax and lost foam), the pattern is used once only. As we will see, this first dichotomy is of considerable importance in terms of the structure of production costs.

$\left(P_{2}^{1}, P_{2}^{2}\right.$ : hollow or full): The molding methods can be classified according to the fact that the mold cavity is hollow (the pattern is extracted before the casting) or full (foam polystyrene as expandable pattern is gasified by the molten metal during the casting. By taking the place of the expandable pattern, the molten metal fills in the full cavity as it would fill in a hollow cavity).

$\left(P_{3}^{1}, P_{3}^{2}\right.$ : chemical or physical): The molding methods can be further classified according to the kinds of bonding systems used for the stabilization of individual granules of molding material used to form a compact casting mold.

$\left(P_{4}^{1}, P_{4}^{2}\right.$ : simple or complex): finally, both chemical and physical bonding methods can be described as simple (mechanical and inorganic chemical binder) or complex (magnetic field, vacuum and organic chemical binder).

Fourth, each parameter corresponds to a given level of aggregation (or integration). The four levels can be ordered hierarchically, according to the relation between the parameters. For example the stabilization force $\left(P_{3}\right)$ influences the bonding method $\left(P_{2}\right)$, or the nature of the pattern $\left(P_{1}\right)$ influences the nature of the mold cavity $\left(P_{4}\right)$. 


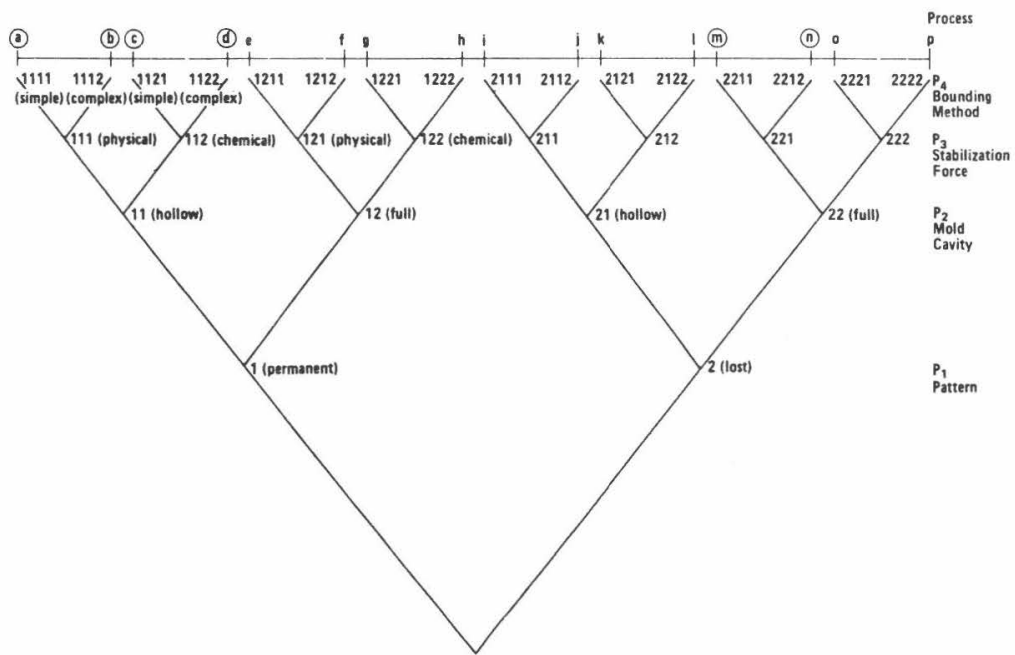

Fig. 1. The morphological space of molding processes (with 4 parameters).

This hierarchical structure allows us to represent the morphological space of molding technology as an "arborescent" scheme (fig. 1), which gives a systematic representation of all possible alternatives to the casting problem.

In terms of graph theory, an "arborescent" structure is a tree with an original node (that is a given point, so that each other vertex can be attained by a path coming from this point). A graph which possesses an original node is "quasistrongly connected" (for all pairs $x, y$, there exists a vertex $z(x, y)$ from which start at once a path to $x$ and a path to $y$ ).

These properties will be used in the following to evaluate the morphological distances between the different processes.

Let us now introduce some precisions.

(a) The set of combinations of the morphological tree does not describe the technical processes but it shows the combinations of parameters which constitute those processes. In other words, this first approach is concerned with the "deep structure" and not with the "surface structure of technical knowledge" [17]. Then the molding processes (corresponding to the "surface structure") can be located above each terminal vertex (i.e. combination of parameters) of the graph. Below, we describe the main processes which correspond to each combination of parameters:

$a$ : green sand, synthetic sand, bentonite;

$b$ : magnetic molding process, $\mathrm{V}$ process, effset; $c$ : cement, silicate $\mathrm{CO}_{2}$, furan, alkyd, liquid sand, etc;

$d$ : oil sand, croning, hot box, thermoshoc, etc;

$e:)$

$f:$

$g:$ self-contradictory solutions

$h:$ )

$i:$

\begin{tabular}{l|l}
$j:$ & precision foundry as lost wax processes \\
$k:$ &
\end{tabular}

$l:$ :

$m$ : lost foam, polylok

$n$ : magnetic molding process, replicast, replicast CS

$o:$ evaporative casting process

$p:$ self-contradictory solution

(b) The MA applied to molding technology results in 16 distinguishable combinations, for four parameters, although some are self-contradictory: thus some states of one parameter are not compatible with some states of another parameter. Therefore, the combinations $(e, f, g, h)$ are impossible, given the incompatibility between the permanent nature of the pattern and the full nature of the mold cavity. $(p)$ is also self-contradictory. Once the impossible solutions are eliminated from consideration, the morphological model can be used to evaluate the actual technological development of the considered industry.

(c) We are not yet capable of formulating any 
conclusion concerning the economic value of each combination, or their relative contribution in the output (market share) of the sector. The goal of the MA is instead to provide a comprehensive definitional structure of the process technologies available and a taxonomy of their evolution. Economic data and analysis of market shares of different processes are thus no longer based on ad hoc considerations, but on the results of a comprehensive structural analysis methodology.

(d) Thus, the profits of the MA are the following. The first one consists in the possibility to examine if the technological development of an industry does recover the totality of its morphological space. Therefore, the whole morphological space will be divided into known and unknown (terra incognita) subspaces. The second interest of the MA consists in the possibility of defining rigorously what technologies are in competition.

\section{The technologies in competition}

\subsection{A morphological procedure for identifying com- peting technologies}

The specification of rival technologies includes two notions.

(a) A notion of substitutability; two technologies which do not assume the same basic function cannot be considered as being in competition. This basic function refers both to a dimensional criterion (for example mass-production) and to a qualitative criterion (for example a given degree of complexity of products). According to this first constraints, we can conclude that five solutions ( $i$, $j, k, l, o)$ are inadequate for mass-production and consequently not in competition. On the contrary, the solutions $(a, b, c, d, m, n)$ are substitutable. Indeed those methods are devoted to mass-production, for a common minimum level of the complexity of the product.

(b) A notion of morphological distance; it is essential to define theoretically a technological change, either as an improvement of an existing technology or as the emergence of a rival technology. In this perspective, we will consider that competing technologies are separated by a given morphological distance $(M D)$ which is estimated below. The $M D$ will be calculated on the following graph, called graph $G$, from which the selfcontradictory solutions are eliminated, as well as

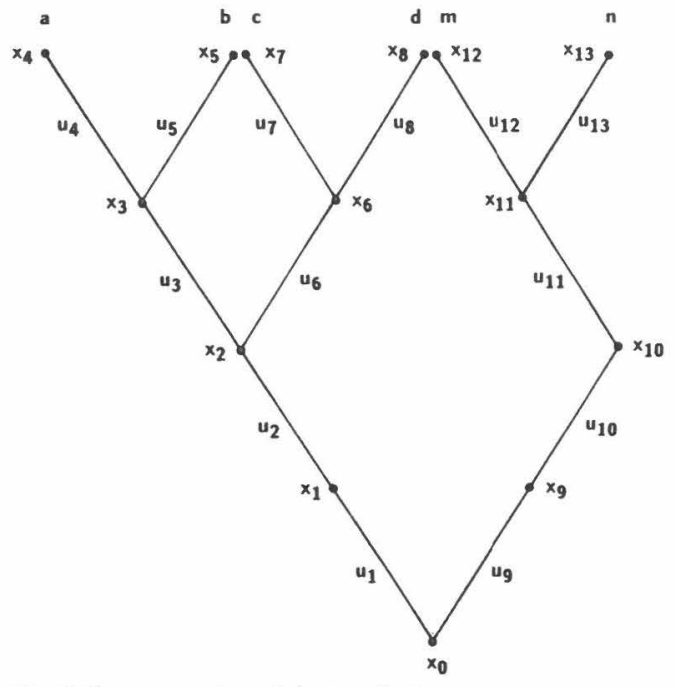

Fig. 2. Representation of the graph $G$.

the solutions which are inadequate to mass-production (fig. 2).

$G=(X, U)$, is the couple, constituted: First, by a set $X=\left(x_{1}, x_{2}, \ldots, x_{n}\right)$, and second by a family $U=\left(u_{1}, u_{2}, \ldots, u_{m}\right)$ of elements of the cartesian product $X \times X=((x, y) / x \in X, y \in X)$.

This graph verifies the properties of an arborescent structure as discussed above. In order to estimate the MD between two points in the space (i.e. the number of parameters differing from one another in two configurations), we use the notion of path.

A path of length $q>0$ is a chain of a particular type: $\mu=\left(u_{1}, u_{2}, \ldots, u_{q}\right)$, such as for each arc $u_{i}$ (with $i<q$ ) the terminal extremity of $u_{i}$ coincides with the initial extremity of $u_{i+1}$. MD between two terminal vertices (two processes) is the length of the corresponding path $\mu$, i.e. the number of arcs of the sequence:

$M D(a, b)=\mu^{2}=\left(u_{4}, u_{5}\right)$

$\operatorname{MD}(a, c)=\mu^{4}=\left(u_{4}, u_{3}, u_{6}, u_{7}\right)$

$M D(a, m)=\mu^{8}=\left(u_{4}, u_{3}, u_{2}, u_{1}, u_{9}, u_{10}, u_{11}\right.$, $\left.u_{12}\right)^{4}$

We must then define a critical distance, according to which some technological changes occur

${ }^{4}$ On account of the hieràrchical character of the graph (as demonstrated in the discussion above), the estimation of the value of each arc should have to take into account a coefficient of ponderation reflecting its proximity to the original node. 
inside a morphological neighbourhood (for example $b$ with reference to a), while some others occur outside and can thus be defined as the emergence of a rival technology ( $\mathrm{m}$ with reference to a). In the theory of the quasi-strongly connected graph, this critical distance is given by the notion of radius of the graph $G$.

The directed distance $d\left(x_{i}, x_{j}\right)$ is the length of the shortest path from $x_{i}$ to $x_{j}$. The "associated number" of a vertex $x_{i}$ is $e\left(x_{i}\right)=\max d\left(x_{i}, x_{j}\right)$ with $x_{j} \in X$ and $x_{j} \neq x_{i}$. The "center" is a vertex $x_{0}$ with a minimum associated number. $e\left(x_{0}\right)$ is called "radius" of graph $G$ and is noted $p(G)$. On fig. $2, p(G)=4$. Thus, $(M D \leqslant 4)$ defines a morphological neighbourhood and $(M D>4)$ defines a technological breakthrough (rival technologies).

Our morphological procedure results in the identification of two competing technologies and consequently of two morphological neighbourhoods: sand molding processes (SM process), corresponding to the combinations of parameters $(a, b, c, d)$ and gasifiable pattern processes (GP process), corresponding to $(m, n)$.

According to Metcalfe and Gibbons [10], we find it helpful

to analyse technology at two conceptual levels. In terms of artifacts, the products and processes of production which firms reveal in the market place; and in terms of the corresponding knowledge bases, the ideas, concepts and modes of enquiry which are necessary to generate a particular revealed performance.

We consider thus that while the displacement of a firm inside a morphological neighbourhood $(a \rightarrow b$ or $m \rightarrow n$ ) implies for it only a change of artifacts, the incorporation of a rival technology $(a \rightarrow m)$ implies both changes in the artifacts and its knowledge base.

Let us now introduce some considerations concerning the economics of technological competition in case of molding technology.

\subsection{Economics of technological competition}

We attempt to characterize economically the technologies in competition (SM process vs GP process) at two complementary levels.

\subsubsection{Technical complexity and simplification of the operating methods}

This first level refers to one of the characteris- tics of technical evolution [5]: as technological processes become more complex, operating methods tend to become more simplified. According to Pratten [13] and Foray [5], the main steps of production, respectively used both in SM and GP processes are as shown in fig. 3.

Thus, the generation of the GP process results in an extreme simplification of the operating methods:

The GP process involves investing an injection molded foamed-polystyrene pattern in a free flowing magnetizable molding material. Immediately prior to pouring, the molding material is rigidized by a powerful magnetic field. During casting, the polystyrene pattern volatizes in the face of incoming metal stream which occupies the void left by the gasified pattern. Shortly after the casting has solidified, the magnetic flux is switched off and the flask containing the casting is taken to the knockout station [9].

But this simplification is associated with increasing technical complexity: a low level of complexity (SM process) corresponds to an extreme complication of the operating methods, while a high level of complexity (GP process) corresponds to an extreme simplicity of the operating methods. The history of the casting industry's technical progress clearly shows a process both of increasing technical complexity and of a simplification of operating methods.

\subsubsection{Structure of costs and economies of scale}

The second level of characterizing competing technologies deals with the question of costs structure and economies of scale. Importance of learning in the finishing processes and relatively minor learning in the preparation and pouring processes are common features which affect the conditions of economies of scale in both MS and GP technologies:

Changes in products should not materially affect the cost of preparing sand and iron melting, provided that the type and quality of iron used do not change, nor should it affect the cost of pouring the iron into the moulds, but it does affect the finishing processes. A switch of products involves operatives performing different operations which are subject to a learning 


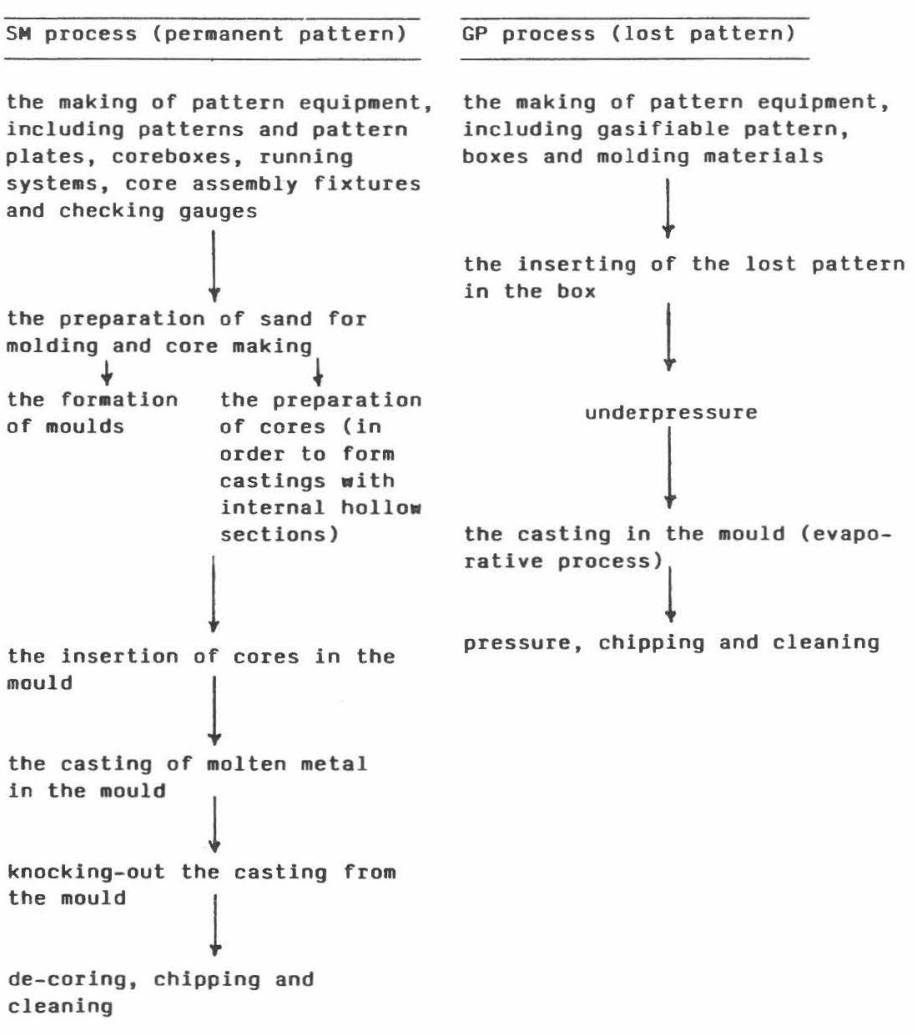

Fig. 3. The main operations of production used in SM and GP processes.

process and it is claimed that this learning process is associated with the length of individual production runs, rather than the overall output of a product, although this also has a slight effect. It is not only the time lost in learning a new routine but also the decline in quality that increases unit costs [13].

On the contrary, the problem whether the pattern costs are included either in the initial costs or not, represents a key-discriminatory feature between the competing technologies: in the case of SM processes, one of the main economies to be achieved by increasing output of individual castings is the spreading of pattern costs. The effects of spreading pattern costs are illustrated by the data shown in table 1: the higher the relative importance of pattern costs (the cost of a wooden pattern would be about 25 percent of the cost of a metal pattern) the more crucial is the search for mass-production.

On the contrary the cost of a lost pattern cannot be included in the initial costs. Given that a lost pattern is utilizable for a unique casting, it is necessary to produce as many patterns as products. Therefore there is no direct relationship between the pattern cost per unit and the importance of the run, so that the decrease of the pattern cost per unit can be achieved only by the rationalization of the production of patterns. Before those rationalization efforts, the GP process is thus inadequate to the mass-production (fig. 4a). This flat pattern costs per number of castings explains both the limits of the GP process and its competitive advantage over the SM process for the production of small batch sizes. At this period, the GP process was diffused inside a small market niche only, where it was in competition with the 
Table 1

Pattern cost as initial cost in SM process

\begin{tabular}{|c|c|c|c|}
\hline Number of castings & 1 & $\begin{array}{l}10 \\
£\end{array}$ & 100 \\
\hline \multicolumn{4}{|c|}{ Simple conveyor casting (wt 7 lbs) } \\
\hline Pattern cost & 3.00 & 3.5 & 22.0 \\
\hline Production costs per unit & 0.53 & 0.45 & 0.35 \\
\hline Total cost per unit & 3.53 & 0.80 & 0.57 \\
\hline \multicolumn{4}{|l|}{ Gear box casting (wt 2 cwts) } \\
\hline Pattern cost & 100 & 110 & 140.0 \\
\hline Production costs per unit & 15.0 & 13.5 & 12.0 \\
\hline Total cost per unit & 115.0 & 24.5 & 134. \\
\hline \multicolumn{4}{|c|}{ Tailweight casting (wt $15 \mathrm{cwts}$ ) } \\
\hline Pattern cost & 60 & 75 & 110.0 \\
\hline Production cost per unit & 75 & 65 & 55.0 \\
\hline Total cost per unit & 135 & 72.5 & 56.0 \\
\hline
\end{tabular}

The estimates of costs given in this table were obtained from a firm which makes engineering castings. The figures for pattern costs were lower than those quoted by other firms, and it should be noted that patterns frequently cost several thousand pounds.

Source: Pratten [13].

SM process for unit production of very complex and large products. After the rationalization of the production of patterns (fig. 4b), the GP process diffused also for mass-production: the stake of competition between SM and GP processes (i.e. the size of the market to be conquered) becomes more and more important.

Thus, it is indeed the cost structure characteristic of the GP technology that constitutes the criterion of the periodization of the diffusion process: our diffusion study will analyse these two successive steps of market penetration.

\section{The dynamics of competing technologies in the morphological space of the casting industry}

We attempt now to describe the historical sequence in the emergence of the initial processes (those which are generated by a new combination of parameters) as well as the subsequent dynamics of improvements. Therefore the classes of processes $(\mathrm{a}, \mathrm{b}, \mathrm{c}, \mathrm{d}, \mathrm{m}, \mathrm{n})$ which corresponded to the combinations of parameters on the graph $G$ will correspond now, according to our estimation of the critical morphological distance, to two technological trajectories, respectively $(a, b, c, d$ : SM processes) and ( $m, n$ : GP processes). While our first approach (graph $G$ ) was oriented toward the definition of the "technical possibilities" (the "frontiers") in the foundry industry, this second approach attempts to describe the technological trajectories which have actually evolved in the industry. The gap between the technical possibilities described by graph $G$ and the actual technological development will be explained then in terms of diffusion theory.

\subsection{Procedure of historical analysis}

We make use of a data base consisting of 50 innovations in the foundry industry, with their

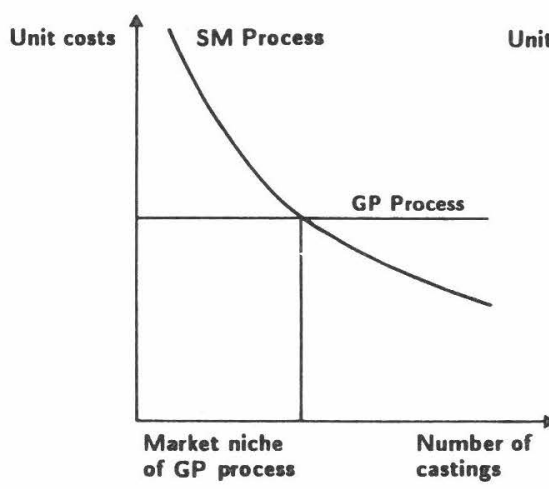

a) Gasifiable Pattern Process growths in limited market (small batch size production)

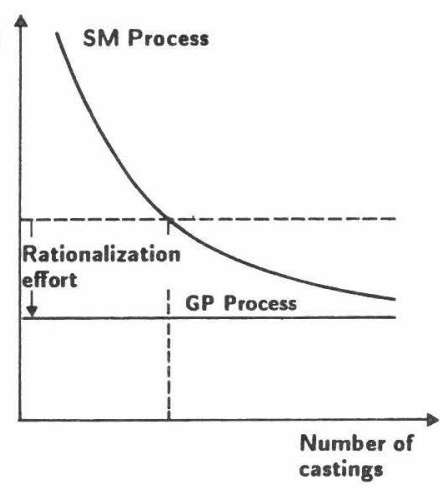

b) Gasifiable Pattern Process diffuses into mass production

Fig. 4. Evolution of structure of costs and the two successive steps of market penetration for the GP process. 
technical description and a historical dating of their diffusion. At the beginning of the first period (1945) three processes coexisted (green sand, cement, oil sand) belonging to a common technological trajectory (SM process). All these processes are based inherently on a permanent pattern (parameter $P_{1}$ on fig. 1) but specified by different bounding methods or stabilization forces $\left(P_{3}\right.$ and $\left.P_{4}\right)$. Then we follow the improvements within that technological trajectory as well as the creation of a new one (GP processes based on lost pattern). In comparison with figs. 1 and 2, our investigation is concerned now with the "surface structure of technical knowledge" while the "deep structure" will serve to locate the ramifications on the graph. Contrary to figs. 1 and 2, fig. 5 is not definitive. Its principle of construction gives the possibility of a parallel evolution between the system of observation and the observed reality.

\subsection{Describing the dynamics of technology (fig. 5)}

According to the experts, since around 1950 the number of improvements in foundry technology has expanded exponentially. During the last decade this has led to the "age of foundry technology".

It may be that foundrymen would agree to dating the appearance of this 'age' from the development of the shell molding process by J. Croning in the late 1940's. Since that time, a broad variety of casting production processes have been developed, introduced, and used in the foundry industry. The result has been a remarkable rationalization of production, applying to permanent and to lost molds [19].

During this "age of foundry technology", we discern three key features:

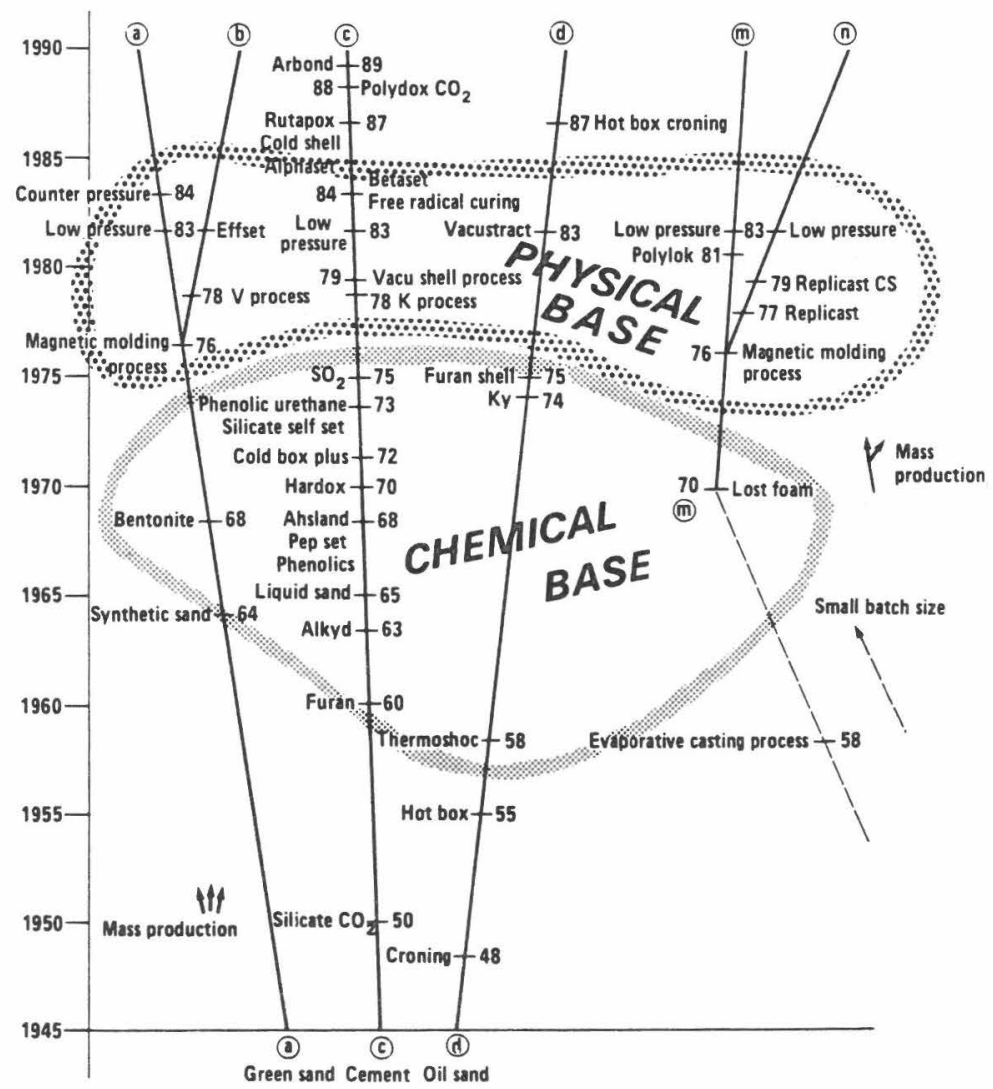

Fig. 5. Trajectories of molding processes and cluster of innovations (1945-1989) 
- Clustering of chemical based innovations between 1955 and 1975: lost molds predominantly or completely bonded by chemical means (development of the existing trajectory by changes at levels of parameters $P_{3}$ and $P_{4}$; see fig. 1);

- emergence of a rival technology: GP process (creation of a new trajectory by changes at level of parameter $P_{1}$ );

- clustering of physical based innovations between 1970 and 1985: lost molds predominantly or completely bonded by physical means (development of the existing trajectories by changes at levels of parameters $P_{3}$ and $P_{4}$ ).

\subsubsection{The first cluster of innovations}

Figure 5 shows a first cluster of innovations during the period 1955-1975. This cluster was oriented toward the use of chemical means for the stabilization of the mold. Originally the chemical methods were used by applying cement, $\mathrm{CO}_{2}$ gas, oil sand and shell molding (Croning) (see bottom of fig. 5). Then improvements in the application of inorganic and organic binders determined a cluster of innovations (furan, alkyd, phenolics, pep set, bentonite, thermoshoc, etc.). According to the $\mathrm{MA}$, these technological changes cannot be considered as the emergence of a rival technology (all morphological distances are inferior to the radius of the graph $G$ ). Since 1958, the GP process was used, but, given its specific cost structure, discussed above, it was devoted to small batch size and thus not in competition in mass-production:

Use of the process began in the production of large short run castings such as automotive die castings. In this application bonded sand was normally used and the pattern were handmade from sheets of expandable polystyrene [2].

This initial version of the GP process corresponded to the combinations of parameters $\mathrm{o}$, on figs 1 and 2 .

\subsubsection{The emergence of a rival technology}

In 1970, significant improvements concerning the GP process occurred. In particular, rationalization in the production of lost patterns (pre-expansion and molding processes of expandable polystyrene) made this process adequate for mass-production, so that the GP process (combination of parameters $\mathrm{m}$ ) became substitutable to all existing SM processes (a, c and d):
The future of the gasifiable pattern process appears to be in large production runs using molded polystyrene patterns in unbonded sand. This is in contrast to its original use which was in the production of large short run castings [2].

According to the MA, this technological change can be considered as the emergence of a rival technology, given the substitutability of the processes and the morphological distance between those processes (superior to the radius of the graph $G)$.

\subsubsection{The second cluster of innovations}

The cluster of physical based innovations (use of vacuum and magnetic fields) occurred since 1975 , the year of the first industrial application of magnetic molding. Magnetic molding was introduced both to SM processes (magnetic molding, V process) and to GP processes. ${ }^{5}$ The new technical principle of the gasifiable pattern process (replicast, replicast CS) was the following:

It consists of a massproduced, injectionmolded, polystyrene-foam pattern encased in a free-flowing magnetizable material, free of any substantial bonding additives, and contained within a one-part flask. The pattern is enveloped by the molding material simply by pouring the free-flowing material around it. The magnetic field is then switched on and maintained during pouring and as long as necessary during the solidification of the melt. Finally, the magnetic field is switched off and the casting can easily be removed from the molding-material which has again become free-flowing [19].

The importance of this second clustering can be " related with two additional features: the extension of vacuum principles to the chemical based processes (vacushell, vacustract) and to the casting processes (counterpressure, low pressure).

In conclusion, the technological structure of the industry is going (via post-innovations) to occupy

\footnotetext{
${ }^{5}$ With respect to the SM trajectory, the technical improvements which occur during this last period give us a good illustration of the so called "sailing ship effect". However, improvements in the SM trajectory did not affect the longterm competitive position of SM technologies compared to the technological improvements of the GP process (cf. discussion in section 6).
} 
nearly the totality of its morphological space. However, the situation in individual countries is entirely different.

5. FRG, France: national patterns of molding process trajectories and global market evolution

Figure 6 shows the differences between the technological structures of France and the FRG: while the German structure occupies the totality of the morphological space, the French structure leaves a large part of it uncovered.

Figure 7 supports this statement. Two important features can be observed and commented on. First, the foundry industry follows a very similar way of evolution in terms of output volume in France and in the FRG. A period of saturation and contracting markets appeared after a period of growth and relatively expanding markets. In each case, the turning point occurred in the early 1970s. Second, since 1960, the GP process started to diffuse in the FRG while in France it was locked-in in a very minor market share position and never took off in a diffusion phase.

Figure 7 also shows that in the case of the FRG, the diffusion pattern of the GP process was not influenced by the global fluctuation (i.e. decline in the output volume) of the industry and that the output figures of the GP process were apparently not affected by the strong fluctuations
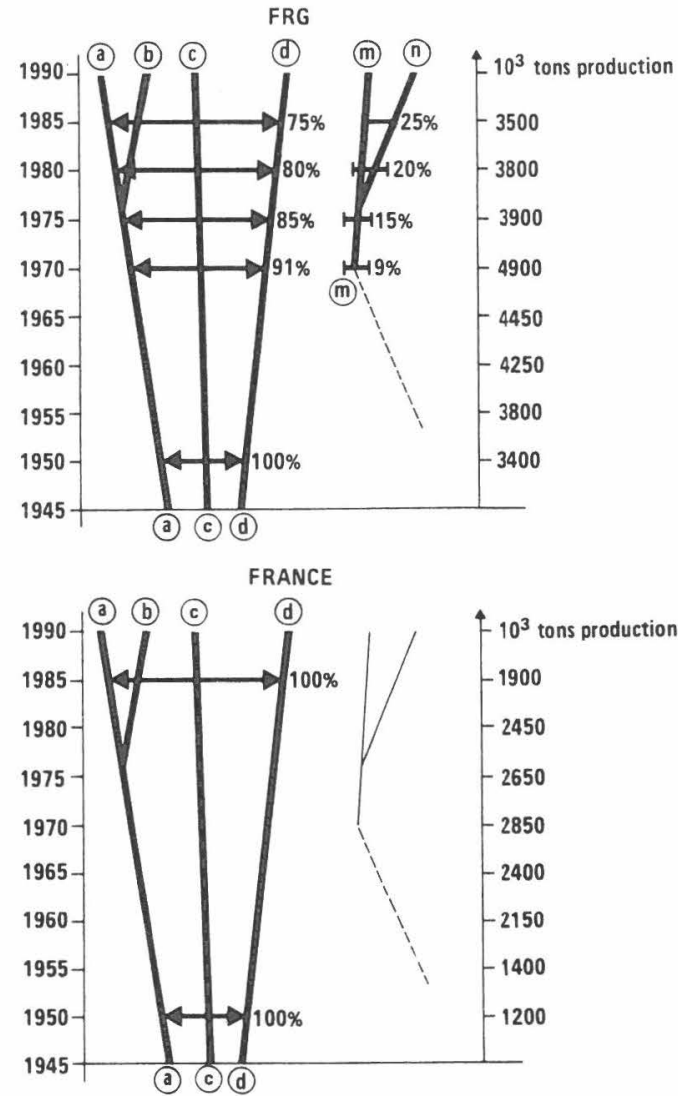

Fig. 6. National patterns of the molding process trajectories (mass-production).

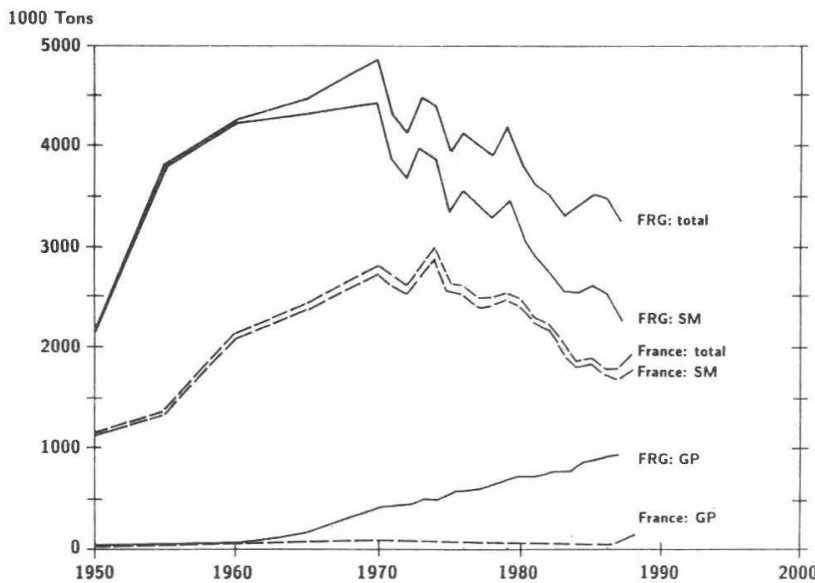

Fig. 7. FRG and France: Casting by process. 
in the total market volume. The evolution of the SM process on the other hand appears to follow closely these global market fluctuations.

Thus we conjecture that in diffusion research it is important to differentiate two important different situations with respect to the evolution of the market technologies compete in. In a first case, where the market expands rapidly, diffusion is taking place by differential growth rates in the incorporation into the capital stock, i.e. changing relative market shares are the result of one technology growing faster than an other one. This is in sharp contrast to diffusion of a technology in a saturating, even declining market, as in our case. We maintain that under such market conditions, effective diffusion calls for a higher comparative advantage than in the first case.

It is noteworthy to consider the situation in the FRG as shown in fig. 7. Despite strong market fluctuations the evolution of output figures of the GP process are very regular, i.e. not affected by short-term business cycle variations in market volume. Conversely the SM process takes the full burden to act as "swing supplier", i.e. responding to demand fluctuations.

It is our contention that this different behaviour towards demand fluctuations is indicative for a high comparative advantage differential between the two processes in the FRG.

In the next section we discuss what are the possible sources of those differences between the national patterns of development.

\section{In search of specific factors of diffusion in France and the FRG}

Our above discussion of the morphological structure of the technological trajectories permits us to conclude that prior to 1970 , the GP process (which was corresponding to the combination of parameters o, figs 1 and 2) could compete only for the casting of small batch size. In a second period, after a technological breakthrough concerning the conditions of production of lost patterns, the GP process (which was corresponding then to the combinations of parameters $m$ and $n$ ) could effectively diffuse also in mass-production and compete with the SM process. Thus, in order to explain the differences between the national patterns of diffusion, it is necessary to divide the adoption process of GP technology into two phases: The diffusion into the first market niche of complex, small series-production; and the subsequent diffusion into the mass-production market.

Figure 9, which will be discussed in section 7, illustrates the extreme rapidity of the substitution process in the FRG during the first phase. As driving forces, we shall examine the dynamics of demand structure of the casting industry and the differential in the specific value-added between the two competing technologies (i.e. a first order approximation for their relative profitability). Then, we shall discuss the influence of the first period's diffusion pattern on the outcome of competition in the second period.

\subsection{Dynamics of demand structure in the first market niche}

A first driving force relates to the evolution of the demand corresponding to the market niche of complex, small series-production. Thus we conjecture that this highly specialized market expanded rapidly in the FRG in the early 1960s (not the case in France). This specific expansion played (as discussed in section 5) a significant role as a factor to explain the rapid substitution in the case of the FRG. The development of this argument is however. seriously hampered by the absence of relevant statistics prior to 1970 .

6.2. The differential of the specific value-added between the two competing technologies: take-off in the first period in the $F R G^{6}$

Figure 8 illuminates the role of the value-added differential as a factor of explanation of the national patterns of diffusion. It shows the strong character of the differences between the relative value-added, in particular during the first phase of diffusion: low level (factor 1,1) in France could explain the relative disinterest of the French firms for the new process.

${ }^{6}$ Clearly the nominal value-added differential as illustrated in fig. 8 should be presented in real terms. However estimation of real price deflator faces the difficulty that the structures of the market and product are changing (as demonstrated in the discussion above) and are consequently not reflected appropriately in the index of price indicators published by the industry. 


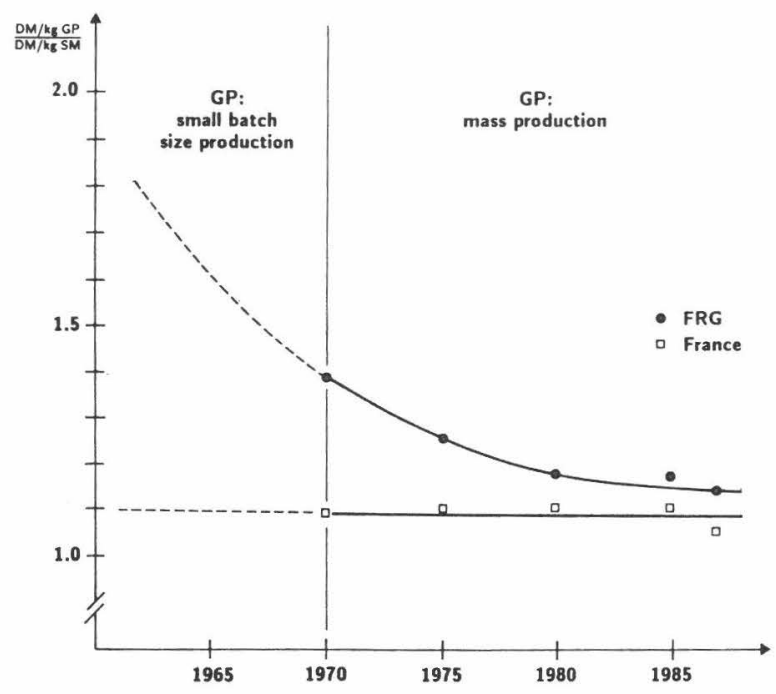

Fig. 8. FRG and France: Relative value-added (profitability) by casting process.

Further, the evolution of the relative value-added in FRG $(1,7 \rightarrow 1,1)$ can be explained by the existence of two phases in the diffusion process: during the first phase, the market niche is concerned with complex, small series-production and the comparative economic advantages of the GP process are stronger than during the second phase of diffusion where it approaches the value-added ratio of mass-production. Thus, the differential represents a first explanation for the GP process's rapid saturation of -its first market niche in the FRG. One question remains to be answered. How did the first period's diffusion pattern in the FRG influence the outcome of competition in the second period?

\subsection{Knowledge accumulation and learning process during the first period of diffusion in the FRG}

Thus during the first period of diffusion, the technology was rapidly adopted in the FRG, in spite of the fact that its adoption caused a strong technological breakthrough for the innovative firms. What is fundamental here is what occurred to some extent "underground" in this first diffusion phase. This pattern of diffusion generated a process of accumulation of knowledge, through adequate institutional arrangements: a technological foundation was created in Ludwigshafen am Rhein, with strong participation by German firms (foundries and chemical enterprises). Research programs were oriented towards the improvement of the use of polystyrene patterns to produce metal castings and the systematical generation of minor innovations, required for the industrialisation of the GP process. For example:

Research conducted reveals that top, side or bottom gating can be used with success for the operation of casting. The researchers used high speed cine (motion picture) film to record the actual pouring of GP process molds as viewed through a clear silica glass panel. In most cases, the films showed the metal progressing in a laminar flow from the ingate, but in several cases... [2].

Thus, optimal pouring rate, adequate density of polystyrene, etc. are systematically investigated. After the seminal conception of the process (which can be interpreted as a jump in technical knowledge), research programs were systematically conducted in the FRG, with respect to the technological problems which successively occurred during the diffusion phase (production of the corresponding scientifical knowledge) [7]. Thus, knowledge was accumulated during the first phase of diffusion through an adequate institutional arrangement. More generally, this initial diffusion in a higly specialized market permitted the GP process to access, for the first time, those mechanisms 
related to the increasing returns to adoption (cf. footnote 3 ), principally learning by using, scale economies in production and informational increasing returns, all the while being "protected" by the high value-added differential. These technological accumulation and learning processes can be viewed as an important factor in the technological breakthrough (adaptation to mass-production) of 1970.

On the basis of the features discussed above, we will try to comment on the diffusion trajectories of the GP process in the FRG.

7. Diffusion trajectories of the GP process in the FRG and lessons for exiting from a "lock-in" situation

The key features of GP process diffusion in the FRG are the following.

Between 1958 and 1970, in spite of the fact that the introduction of the GP process caused a strong technological breakthrough (artifacts and knowledge base) for the firms which committed themselves into this new trajectory, the process is rapidly adopted in the small batch size market niche (fig 9): the high value-added differential (fig. 8 ) is the main factor which explains the pattern of market penetration during the first phase of diffusion in the FRG.

In 1970, the adaptation of the GP process to mass-production was realized in the large foundries of the German automotive industry.

The German and French examples are quite instructive with respect to exiting from a "lock-in" situation. The "lock-in" theory allows us to explain how a new and intrinsically superior technology may be impeded from supplanting an older technology. According to Rosenberg and Frischtak [15, p.147]: "New inventions are typically very primitive at the time of their birth. Their performance is usually poor, compared to existing (alternative) technologies as well as to their future performance."

Thus, when a new technology is introduced in its initial (and therefore primitive) form, it has virtually no chance of imposing itself, even if the old technology is "inherently inferior". The latter has profited from its monopolistic period to entrench itself materially (via technological interrelatedness) and intellectually (via sui generis evaluation norms) as the dominant productive paradigm. In this respect, our case study displays the crucial nature of an initial diffusion in a highly specialized market. In this first period the new technology, "protected" by a high value-added differential, may improve within a "quasi in vitro" development, so to speak. Thus shielded, the new technology may acquire industrial properties via the mechanisms related to the increasing returns to adoption, gradually armouring itself for competition. Between 1950 and 1970, the GP process improved in a virtually underground fashion in the FRG; it was later able to enter the main competition arena under auspicious conditions. Having missed the first phase, France is now missing the second one.

A formal analysis of the diffusion trajectories of the GP process in the FRG through two successive market niches, small batch size production prior to 1970 and equally mass-production thereafter is however seriously hampered by the absence of relevant production statistics. For the diffusion trajectory within the first market niche of small batch production we assumed a constant volume of complex castings being produced in small series in the FRG in the period prior to the mid-1970s to calculate the fractional market share of the GP process within this limited market niche. For the second phase of diffusion we calculated the diffusion trajectory on basis of the fractional share in total (tonnage and value) output, based on the conclusion of the morphological analysis, which has yielded that the GP process is in effective competition also for mass-production.

Table 2 and fig. 9 summarize the quantification of the diffusion trajectories in the case of the FRG, based on a simple Fischer-Pry [4] type of technological substitution model (the properties and underlying assumptions of this now classical model will not be repeated here; details on the estimation algorithm used can be found in Grübler, Nakicenovic and Posch [8]). In order to increase the analytical resolution of the formal

\footnotetext{
${ }^{7}$ The use of the Fischer-Pry model to describe the diffusion of the GP process in two distinct periods is based on the argument that the theoretical structure of this model is appropriate for taking into account this mix between a phenomenon of continuity and a two-period analysis. However, the question of the use of other types of diffusion models (threshold/probit model) is open.
} 
Table 2

Two phases in the diffusion of the GP process in the casting industry of the FRG: diffusion model parameters ${ }^{\text {a }}$

\begin{tabular}{|c|c|c|}
\hline & $\begin{array}{l}\text { Fraction of GP } \\
\text { in tonnage } \\
\text { output }\end{array}$ & $\begin{array}{l}\text { Fraction of GP } \\
\text { in output value }\end{array}$ \\
\hline $\begin{array}{l}\text { Phase 1 } \\
\text { (small batch } \\
\text { size market } \\
\text { niche) }\end{array}$ & $\begin{array}{l}\Delta t=13,1 \mathrm{yrs} \\
(14,74) \\
t_{0}=1967,8 \\
(14,74) \\
\text { Period: } 1960-1977 \mathrm{r} \\
n=10 \\
R^{2}=0.965\end{array}$ & o data available \\
\hline $\begin{array}{l}\text { Phase } 2 \\
\text { (total market } \\
\text { incl. mass- } \\
\text { production) }\end{array}$ & $\begin{array}{l}\Delta t=52,4 \mathrm{yrs} \\
(45,76) \\
t_{0}=1997,7 \\
(45,99) \\
\text { Period: } 1970-1987 \\
n=18 \\
R^{2}=0.992\end{array}$ & $\begin{array}{l}\Delta t=61,58(17,70) \\
t_{0}=1997,9(17,88) \\
\text { Period: } 1970-1987 \\
n=18 \\
R^{2}=0.991\end{array}$ \\
\hline \multicolumn{3}{|c|}{$\begin{array}{l}\Delta t=\text { diffusion parameter, time in years to grow from } 10 \text { to } \\
90 \% \text { market share } \\
t_{0}=\text { inflection point ( } 50 \% \text { market share), time of maximum } \\
\text { growth rate of market shares } \\
\text { Values in parentheses refer to } t \text { statistics of estimated diffu- } \\
\text { sion model parameters. }\end{array}$} \\
\hline
\end{tabular}

description of the second diffusion trajectory, we have used in addition to output tonnage equally the output value (in current DM) by casting process in the period since 1970 (data source: Deutscher Giesserei Verband). The estimated diffusion parameters are consistent between the two measures, with the diffusion rate of the GP pro- cess calculated on the basis of output value being around 17 percent slower than on the basis of tonnage figures.

Consistent with the differential in the specific value-added (i.e. DM per $\mathrm{kg}$ of product) between the two process technologies discussed above, we note that the diffusion rate of the GP process into the first market niche of complex, small seriesproduction is significantly by a factor of 4 faster than in the second phase of diffusion into the mass-production market niche. This points to the fact, that in addition to the higher specific valueadded for the GP process technology (at least 1.6 in 1970, and most likely larger in the period before), also other comparative economic advantages, such as the smaller production costs in small series enter as additional influencing factors explaining the rapid diffusion into the first market segment of the GP process.

In fig. 9 we show the two diffusion trajectories into the two successive market niches of the GP process. Particularly noticeable is the regular second diffusion pattern since 1970 . In order to illustrate the decisive structural difference in the technological base in the casting industry between the FRG and France, we have contrasted the diffusion trajectory in case of the FRG with the trajectory of the market share fraction of the GP process in France, which appears locked-in at a constant market share fraction below the 2 percent level. Since 1986 however, this share has increased rather rapidly to the present level below

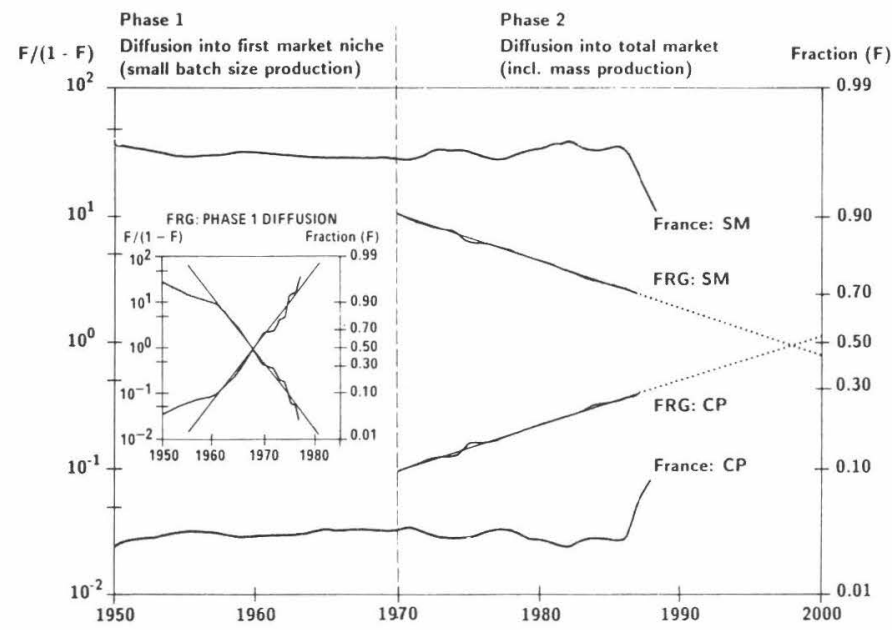

Fig. 9. Two diffusion phases of casting technology in the FRG and lock-out in France. 
8 percent of total casting tonnage in France, which could be a first indication that the GP process might be at the beginning of a similar diffusion take-off as was the case in the FRG.

\section{Conclusion}

Our case study was particularly appropriate to show the advantage of the MA in technological diffusion analysis. Indeed, the MA of the structure of technological trajectories in the casting industry (figs 1 and 2) permits us to avoid misinterpretation concerning the asymmetrical character and discontinuities of the diffusion trajectory of the GP process. On the basis of the morphological space of molding technologies, we can establish that the molding process under consideration (GP) cannot be considered as a unique unaltered artifact throughout the period of diffusion. In fact, there are two diffusion trajectories, corresponding to two combinations of parameters and therefore to two successive market niches. This breakdown into two periods will have thus allowed to formulate a feasible exit process for a "lock-in" situation, by emphasizing the crucial nature of the first period of diffusion, where there occurs accumulation of knowledge and process of learning, within a "quasi in vitro" development. Another important point is that technology continues to change, of course, throughout the diffusion process. In a sense, it is no longer the same technology at the end of the process. However, in another sense it is still the same technology because it is indeed the knowledge accumulated during the first period that is mobilized for competition in the second period.

\section{References}

[1] B. Arthur, Competing Technologies, Increasing Returns, and Lock-In by Small Historical Events, The Economic Journal (March 1989).

[2] M. Bailey, Understanding the Evaporative Pattern Casting Process, Modern Casting 4 (1982)

[3] H. Cameron and S. Metcalfe, On the Economics of Technological Substitution, Technological Forecasting and Social Change 32 (1987).
[4] J.C. Fischer and R.H. Pry, A Simple Substitution Model of Technological Change, Technological Forecasting and Social Change 3 (1971).

[5] D. Foray, Innovation Majeure et Transformation des Structures Productives, Revue Economique 5 (1985).

[6] D. Foray, Innovations Technologiques et Dynamique Industrielle (Presses Universitaires de Lyon, Lyon, 1987).

[7] D. Foray and C. Lebas, Diffusion de l'Innovation dans l'Industrie et Fonction de Recherche Technique, Economie Appliquée 3 (1986).

[8] A. Grübler, N. Nakicenovic and M. Posch, Methods of Estimating S-Shaped Growth Functions: Algorithms and Computer Programs (IIASA, Laxenburg, 1988).

[9] V. Gupta and M. Toaz, New Molding Techniques: A State of the Art Review, AFS Transactions 48 (1978).

[10] S. Metcalfe and M. Gibbons, Technology, Variety and Organisation: A Systematic Perspective on the Competitive Process, PREST (University of Manchester, Manchester, 1988).

[11] V. Milacic and A. Miller, Artificial Intelligence-Morphological Approach as a New Technological Forecasting Technique, International Journal of Production Research 6 (1986).

[12] R. Naro and B. Nelson, Economics of Modern Coremaking Processes, AFS Transactions 61 (1981).

[13] C.F. Pratten, Economies of Scale in Manufacturing Industry (Cambridge University Press, Cambridge, 1972).

[14] N. Rosenberg, Inside the Black Box: Technology and Economics (Cambridge University Press, Cambridge, 1982).

[15] N. Rosenberg and C. Frischtak, Long Waves and Economic Growth: a Critical Appraisal, The American Economic Review-Paper and Proceedings (May 1983).

[16] D. Sahal, Patterns of Technological Innovations (Addison Wesley, 1981)

[17] D. Sahal, Foundations of Technometrics, Technological Forecasting and Social Change 27 (1985).

[18] W. Sulhoff and W. Willis, Automation in the Large Foundry-Progress in the 1970s and an Outlook for the 1980s, AFS International Cast Metals Journal (March 1982).

[19] A. Wittmoser, The New Third Generation of Molding Processes, AFS Transactions 1 (1980).

[20] F. Zwicky, The Morphological Analysis and Construction, in: Studies and Essays, Courant Anniversary Volume (Interscience, 1948).

[21] J. Steindl, Evolution and Technical Progress, in: D. Sahal (ed.), $R \& D$ and Technological Innovation (Lexington Books, Toronto, 1980).

Sources of Data

Die Giesserei Industrie im Jahre: 1975, 1980, 1987, Deutscher Giesserei Verband, Düsseldorf

Die Europäische Giesserei Industrie im Jahre: 1984, 1985, 1986, Comité des Associations Européennes de Fonderie, Köln

Production des Fonderies de France et d'Allemagne Occidentale de 1945 à 1985, Syndicat Général des Fondeurs de France, Paris 